\title{
The Early Career Hydrogeologists' Network (ECHN-IAH): empowering young hydrogeologists at a national and international level \\ L'Early Career Hydrogeologists Network (ECHN-IAH): potenziare il ruolo dei giovani idrogeologi a livello nazionale ed internazionale
}

Viviana Re - Università Ca' Foscari di Venezia, Venice, Italy - echn.iah@gmail.com

Eloisa di Sipio - GeoZentrum Nordbayern, Friedrich- Alexander University Erlangen-Nürnberg, Erlangen, Germany

Keywords: ECHN-IAH, young hydrogeologists, Coolest Paper Award of the Year.

Parole chiave: IECHN-IAH, Giovani idrogeologi, Coolest Paper Award of the Year.

Nato nel 2011, l'Early Career Hydrogeologists Network (ECHN) della International Association of Hydrogeologists (IAH) si rivolge a tutti i giovani idrogeologi (generalmente coloro che si trovano nei primi 10 anni dall'inizio della propria carriera) con l'obiettivo di favorire il loro coinvolgimento nell'associazione e di facilitare l'interazione con i membri senior, supportando così anche la loro crescita professionale.

Fin dalla sua creazione, ECHN, grazie anche al sostegno del consiglio IAH, dei gruppi nazionali e di tutti i soci, ha promosso numerose iniziative rivolte ai membri più giovani, con lo scopo di supportarli nel raggiungimento d'importanti obiettivi, quali la formazione in ambito idrogeologico, la ricerca di lavoro e la cooperazione con tutti i diversi network e commissioni dell'associazione.

A partire dal convegno internazionale del 2012, tenutosi a Niagara Falls, in Canada, ECHN ha inoltre incoraggiato una partecipazione attiva alle attività congressuali da parte dei più giovani. In particolare, specifiche attività che oramai rappresentano parte integrante dei convegni internazionali dell'associazione sono: i) assegnazione di premi per il miglior poster e la migliore presentazione orale; ii) organizzazione di sessioni tematiche in cui esperti idrogeologi forniscono consigli per realizzare una presentazione orale o pubblicare un articolo scientifico; iii) supporto alle attività di mentoring e alla creazione di reti professionali, anche grazie alla serata ECHN organizzata in occasione di ogni congresso internazionale IAH in cui, in un ambiente informale, i giovani idrogeologi possano incontrare i membri senior e discutere del loro lavoro e del loro piano di sviluppo professionale (Fig. 1A); iv) coinvolgimento nell'organizzazione e gestione delle sessioni ufficiali

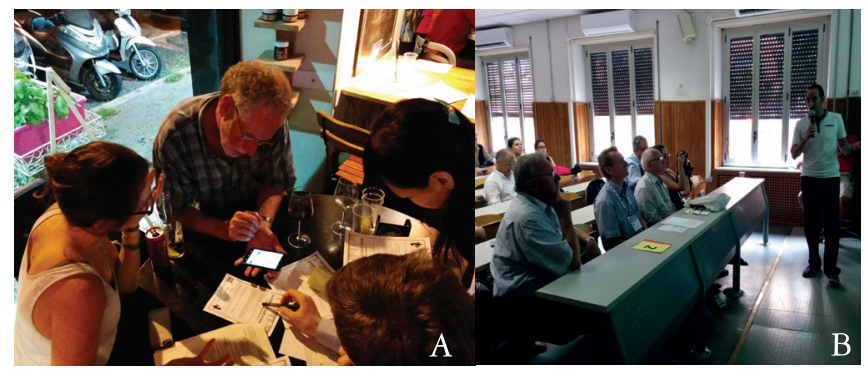

Fig. 1: (A) ECHN Social Function e (B) la presentazione del vincitore de Coolest Paper Award 2015 durante il convegno AQUA 2015 (Roma). Fotografie: Viviana Re.

Fig. 1:(A) ECHN Social Function and (B) presentation of the Coolest Paper Award 2015 winner during AQUA 2015 Congress (Rome). Photo credits: Re Viviana. dei convegni per far sì che i giovani possano fare esperienza pratica affiancati da un membro senior.

Tra le attività più recenti di ECHN a livello internazionale va anche segnalato il Coolest Paper Award of the Year (Fig. 1B), che prevede l'assegnazione da parte dei giovani idrogeologi di un premio per l'articolo scientifico in ambito idrogeologico ritenuto il più interessante e degno di nota (ECHN, 2016). La competizione si svolge interamente via web e le votazioni avvengono tramite un formulario accessibile dal sito ECHN. Il termine cool è stato appositamente scelto per lasciare la più ampia possibilità d'interpretazione, cercando così non soltanto di dare voce ai giovani idrogeologi, che possono esprimere un loro parere riguardo alle pubblicazioni scientifiche inerenti alle acque sotterranee, ma anche permettendo di capire quali siano le tematiche e gli approcci di maggior interesse per le nuove generazioni. Il premio per la terza edizione del Coolest Paper Award of the Year verrà assegnato durante il prossimo convegno internazionale della IAH che si terrà a Montpellier (Francia) dal 25 al 29 settembre 2016.

Il supporto ad ECHN da parte dell'associazione non è mai mancato e recentemente si è anche tradotto in una significativa riduzione delle quote associative per gli studenti e dottorandi, che ammontano ora a 19 (quota studente online) e 29 euro (quota studente che comprende una copia cartacea di ogni volume di Hydrogeology Journal, rivista ufficiale dell'associazione). Questa iniziativa è segno di un importante interesse da parte della IAH al coinvolgimento attivo dei più giovani (IAH, 2016; Fig. 2).

\section{II gruppo ECHN Italiano}

Il 18 giugno 2014, in occasione del convegno FLOWPATH 2014, organizzato dal gruppo italiano della IAH tenutosi a Viterbo, è stata fondata la sezione italiana dell'Early Career Hydrogeologist's Network (ECHN-Italy; Fig 3). L'entusiasmo di un gruppo di ricercatori, dottorandi, assegnisti e professionisti all'inizio della loro esperienza lavorativa come idrogeologi ha portato ad aderire allo spirito e alle iniziative promosse da ECHN International.

È stato creato in territorio italiano un gruppo di riferimento per la condivisione e promozione di iniziative rivolte a chi desidera confrontarsi con altri colleghi all'inizio della loro carriera professionale o accademica su problematiche idrogeologiche, gestione delle risorse idriche e possibilità di lavoro. 


\section{Students:}

Studying groundwater in your degree, or thinking about a career in this area?

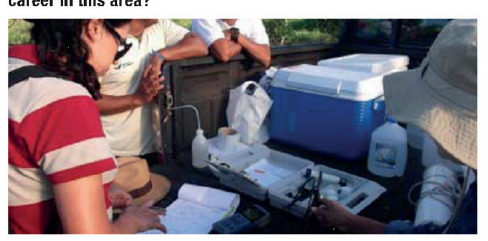

Whether you are looking for information for your latest assignment or are wanting to make the right choices after your course, IAH membership can help. We offer generous student discounts.

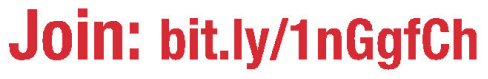

(we'd make you feel most welcome...)

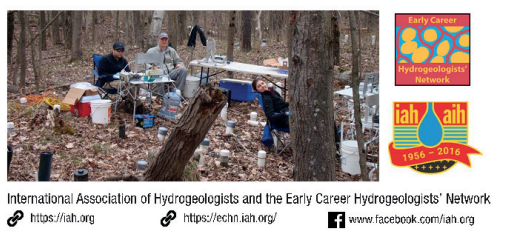

Fig. 2: Volantino ufficiale della IAH per la promozione dell' iscrizione all'associazione da parte di studenti e dottorandi (IAH, 2016).

Fig. 2: Official IAH flier promoting student membership and involvement within the association (IAH, 2016).
La condivisione d'informazioni, la collaborazione con altre organizzazioni, associazioni o istituzioni a livello locale e nazionale e la divulgazione di eventi e manifestazioni a carattere idrogeologico rivolte a chi inizia la sua attività in questo settore costituiscono una delle principali attività di ECHN-Italy (IAH-Italy, 2016).

Il comitato direttivo è costituito ad oggi da:

- Eloisa Di Sipio - GeoZentrum Nordbayern, FriedrichAlexander-Universität Erlangen-Nürnberg, Erlangen, Germany;

- Alessio Barbagli - Scuola Superiore S. Anna, Pisa, Italia;

- Marco Rotiroti - Università degli Studi Milano Bicocca.

Il comitato nazionale vede la partecipazione attiva dei seguenti membri:

- Stefania Stevenazzi - Università degli Studi di Milano;

- Marco Pola - Università degli Studi di Padova;

- Viviana Re - Università Ca' Foscari di Venezia;

- Emma Petrella - Università di Parma;

- $\quad$ Luca Rossi - SINA S.p.A.;

- Maria Filippini - Università di Bologna;

- Mariachiara Caschetto - Università di Roma Sapienza.

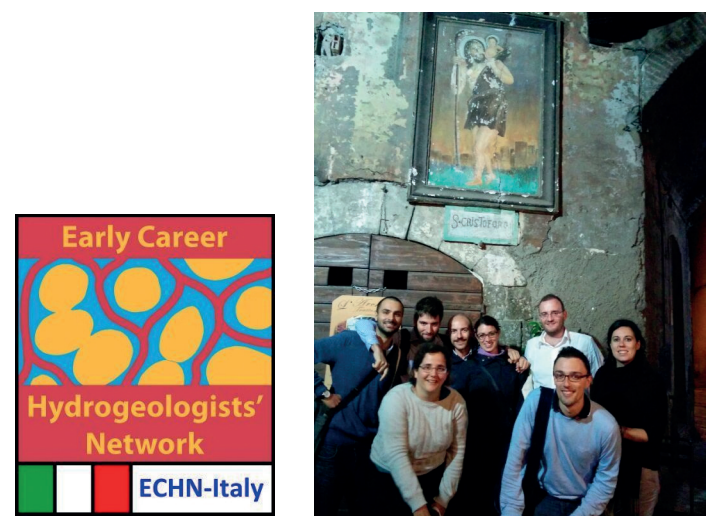

Fig. 3: (A) (A) Logo del gruppo italiano ECHN; (B) Il nucleo fondatore di ECHN-Italy in occasione del convegno FLOWPATH 2014 (Viterbo).

Fig. 3: (A) ECHN-Italy official logo and (B) ECHN-Italy funding members during FLOWPATH 2014 (Viterbo).
Numerose sono le attività organizzate da ECHN-Italy a livello nazionale:

- Incontro rivolto a dottorandi e ricercatori durante FLOWPATH 2014 (18 giugno 2014): Come scrivere una proposta di progetto internazionale MARIE SKLODOWSKA CURIE INDIVIDUAL FELLOWSHIP: preparazione della proposta, processo di valutazione e incontro con vincitore di una borsa MSC, 18-20 giugno 2014, Viterbo;

- Partecipazione al $41^{\text {st }}$ IAH International Congress Groundwater: Challenges and Strategies, 15-19 settembre 2014 Marrakech, Marocco;

- Partecipazione a GeoFuid 2014: Drillings and Foundations, 1-4 ottobre 2014, Piacenza;

- $\quad$ Patrocinio e presenza come conveners alla Sessione VIII La modellazione idrogeologica come strumento per la valutazione della quantità e qualità della risorsa idrica sotterranea, X Convegno Nazionale del Gruppo GIT Geoscienze e Tecnologie Informatiche, Sezione della Società Geologica Italiana, 17-19 giugno 2015, San Leo;

- collaborazione con ECHN International in occasione del $42^{\text {nd }}$ IAH Congress, 13-18 settembre 2015 (Roma): supporto logistico e contributo attivo alla realizzazione delle serate ECHN (Hydro-Cluedo);

- partecipazione come relatori e stand informativo al X Convegno dei Giovani Ricercatori di Geologia Applicata 2016, AIGA 2016, 18-19 febbraio 2016 Bologna;

- co-organizzazione della Sessione IV "Il contributo della modellazione numerica nella caratterizzazione qualiquantitativa delle risorse idriche sotterranee" (co-organizzato da ECHN Italy), XI Convegno Nazionale del Gruppo GIT Geoscienze e Tecnologie Informatiche, organizzato dal GIT - Sezione della Società Geologica Italiana, 13-15 giugno 2016;

Nel prossimo futuro si prevede di continuare la collaborazione con ECHN International e IAH Italy per condividere attività e iniziative rivolte a tutti gli idrogeologi all'inizio della loro carriera e divulgare le varie iniziative mediante social networks e siti web. Pertanto, il gruppo sta lavorando per organizzare un evento in seno al prossimo convegno FLOWPATH 2017 e per coinvolgere un numero sempre maggiore di giovani idrogeologi italiani, in stretta collaborazione con il gruppo internazionale ed il prezioso supporto del comitato italiano della IAH. In questo contesto, proprio per favorire la partecipazione degli idrogeologi all'inizio della loro carriera alle attività dell'Associazione, in particolare aiutando i più giovani in cerca di lavoro, il comitato italiano ha deciso, nell'ultima riunione tenutasi a Roma a maggio 2016, di offrire un contributo a coloro che lasciando lo status di studenti passano da una quota di 19/29 euro a una quota di 70/80 euro. Poiché molti di questi soci passano dal dottorato (quasi sempre pagato) ad una condizione di disoccupazione/precarietà, il comitato ha deciso di offrire per le iscrizioni del 2017 un contributo di 40 euro ad ogni socio che si trovi nelle suddette condizioni. ECHN Internazionale e ECHN-Italy auspicano un sempre maggiore coinvolgimento dei giovani idrogeologi italiani e rimangono a disposizione di tutti i soci per realizzare nuove iniziative di successo a livello nazionale. 